\title{
OS TESTES NÃO-PARAMÉTRICOS G E QUI-QUADRADO
}

\section{E SUAS APROXIMAÇOES}

Marcos A. Antonello Scremin

Curso de Pōs-graduação em Métodos Quantitativos. Departamento de Estatîstica. Centro de Ciências Naturais e Exatas. UFSM. Santa Maria, RS.

Anaelena B. de Moraes Ethur e Maria Emîlia Camargo

Departamento de Estatística. Centro de Ciēncias Naturais e Exatas. UFSM. Santa Maria, RS.

\section{RESUMO}

Neste trabalho é apresentada a procedência e a aproximação de um teste năo-paramétrico alternativo ao teste do Qui-quadrado $\left(\mathrm{x}^{2}\right)$, conhecido na literatura como teste $\mathrm{G}$, mas pouco divulgado. Foi verificada a origem desta estatística através de deduçōes e também a aproximaçăo à estatística do Qui-quadrado, utilizada nos testes de hipóteses.

\section{SUMMARY}

SCREMIN, M. A. A.; ETHUR, A. B. de M. and CAMARGO, M. E., The noparametries tests' $\mathrm{g}$ and chi-square and their aproximations. Ciência e Natura, 13:55-59, 1991.

In this paper we show from where became and the aproach of a alternative to the few published no-parametric test, knowed chis-quare in the literature as G test. By deduction, we research the origin of this statistics and the aproach of the sta tistics of chi-square that is used at the hypothesis tests.

\section{1 - EStatísticA G}

A Estatística G é definida como sendo duas vezes o logarítmo natural da razăo entre a probabilidade da amostra com todos os parâmetros estimados a partir dos dados e a probabilidade da amostra, admitindo-se que a hipótese nula é verdadeira. Esta definçăo origina-se do teste de razăo de verossimilhança, como segue:

Considerando-se $\mathrm{k}$ categorias com probabilidades de ocorrência $p_{1}, p_{2}, \ldots, p_{k} \cdot \operatorname{com} \Sigma p_{i}=1$ para $i=1,2, \ldots, k$. Seja $x_{1}, x_{2}, \ldots, x_{k}$, as frequências observadas naquelas $k$ categorias em $\mathrm{n}$ tentativas, $\operatorname{com} \mathrm{n}=\Sigma \mathrm{x}_{j}$, para $\mathrm{i}=1,2, \ldots, \mathrm{k}$. Entăo a funçăo de verossimilhança é:

$L(p)=\left(p_{1}\right)^{x_{1}} \cdot\left(p_{2}\right)^{x_{2}} \cdot\left(p_{3}\right)^{x_{3}} \ldots\left(p_{k}\right)^{x_{k}}$

Para maximizar $L(P)$, por método de cálculo, deve-se considerar que existe $\mathrm{k}-1$ parâmetros in lependentes, pois $\Sigma \mathrm{p}=1$.

Assim é conveniente escolher $\mathrm{p}_{\mathrm{k}}$ como parâmetro a ser expresso em termos dos parâmetros remanescentes.

Aplicando logarítmos naturais a ambos os membros da Eq.

(1), obtém-se:

$$
\begin{aligned}
& l n L(p)=x_{1} \cdot \ell n\left(p_{1}\right)+x_{2} \cdot \ell \eta\left(p_{2}\right)+\ldots+x_{k} \cdot \ell n\left(p_{k}\right) \\
& \text { Derivando-se a Eq. (2) em relaçăo a p, obtém-se: } \\
& \frac{\ln L(p)}{p_{i}}=\frac{x_{1}}{p_{1}}+\frac{x_{2}}{p_{2}}+\frac{x_{3}}{p_{3}}+\ldots+\frac{x_{k}}{p_{k}}=\frac{x_{i}}{p_{i}}
\end{aligned}
$$


Como só existem k - 1 parâmetros independentes, deve-se eliminar o termo $\frac{\mathrm{x}_{\mathrm{k}}}{\mathrm{p}_{\mathrm{k}}}$. Entăo:

$$
\frac{\ln L(p)}{p_{i}}=\frac{x_{i}}{p_{i}}-\frac{x_{k}}{p_{k}}
$$

Para maximizar é necessário que $\frac{1 n L(p)}{L}$ seja igual a $p_{i}$

zero, ou seja, todas as $\mathbf{k}$ - 1 derivadas parciais desapareçam. Então:

Logo:

$$
\frac{x_{i}}{p_{i}}-\frac{x_{k}}{p_{k}}=\Phi, \text { para } i=1,2, \ldots, k-1
$$

$\mathrm{p}_{\mathrm{i}}=\frac{\mathrm{p}_{\mathrm{k}}}{\mathrm{x}_{\mathrm{k}}} \cdot \mathrm{x}_{\mathrm{i}}$, para $\mathrm{i}=1,2, \ldots . \mathrm{k}$

Aplicando-se o somatório a ambos os membros da Eq. (4) e considerando que $\mathrm{I} p=1$, tem-se:

$$
\begin{aligned}
& 1= \pm \frac{p_{k}}{x_{k}} \cdot x_{i} \longleftrightarrow 1=\frac{p_{k}}{x_{k}}: x_{i} \\
& \text { Como } z x_{i}=n \longmapsto 1=\frac{p_{k}}{x_{k}} \cdot n \text {, entăo: }
\end{aligned}
$$

$\frac{\mathrm{p}_{\mathrm{k}}}{\mathrm{x}_{\mathrm{k}}}=\frac{1}{\mathrm{n}}$

Substituindo-se a Eq. (5) na Eq. (4), obtém-se:

$p_{i}=\frac{1}{n} \cdot x_{i} \longmapsto p_{i}=\frac{x_{i}}{n}, i=1,2, \ldots, k$

testar a hipótese:

Cunsiderando o teste da razăo de verossimilhança para

$$
\mathrm{H}_{\mathrm{o}}: \mathrm{p}_{\mathrm{i}}=\mathrm{p}_{\mathrm{io}}, \mathrm{i}=1,2, \ldots \mathrm{k}
$$

Como năo existe nenhum parâmetro năo específico rê.. nescente quando $\mathrm{H}_{0}$ é verdadeiro, segue-se que a razăo de verossimilhança é dada por:

$$
\lambda=\frac{L(\hat{p})}{L(p)}=\frac{\left(\hat{p}_{1}\right)^{x_{1}} \cdot\left(\hat{p}_{2}\right)^{x_{2}} \ldots \ldots\left(\hat{p}_{k}\right)^{x_{k}}}{\left(p_{1}\right)^{x_{1}} \cdot\left(p_{2}\right)^{x_{2}} \ldots\left(p_{k}\right)^{x_{k}}}
$$

pode-se observar que a Eq. (7) pode ser expressa como sendo a razăo de duas distribuiçóes multinomiais, entăo:

$$
\begin{aligned}
& \lambda=\frac{\frac{n !}{x_{1} ! x_{2} ! \cdots x_{k} !}\left(\hat{p}_{1}\right)^{x_{1}} \cdot\left(\hat{p}_{2}\right)^{x_{2}} \cdot \cdots \cdot\left(\hat{p}_{k}\right)^{x_{k}}}{\frac{n !}{x_{1} ! x_{2} ! \cdots x_{k} !}\left(p_{1}\right)^{x_{1}} \cdot\left(p_{2}\right)^{x_{2}} \cdot \cdots \cdot\left(p_{k}\right)^{x_{k}}} \\
& \text { De (8), tem-se: } \\
& \lambda=\prod_{i=1}^{k}\left[\frac{\hat{p}_{i}}{p_{i}}\right]^{x_{i}}
\end{aligned}
$$


Se as proporçōes p e p̂ forem iguais, entăo a razâo será igual a 1. Quanto maior a diferença entre p e ṕ, com maior intensidade $\lambda$ será diferente de 1 . Isto indica que a razão dessas proba bilidades pode ser usada como estatística para medir a conformidade entre as frequências observadas e esperadas. Um teste baseado em tal razâo é denominado de teste de razầo de probabilidade.

Sendo a frequência esperada $e_{i}=n \cdot \hat{p}_{i}$ e a frequiência observada $x_{i}=n \cdot p_{i}$, entăo:

$$
\hat{p}_{i}=\frac{e_{i}}{n} \quad \text { e } \quad p_{i}=\frac{x_{i}}{n}
$$

substituindo estes resultados na eq. (9), tem-se:

$$
\lambda=\prod_{i=1}^{k}\left[\frac{e_{i}}{x_{i}}\right]^{x_{i}}
$$

Como a distribuiçăo teórica dessa razão é complexa e pouco conhecida, considera-se o seguinte teorema "Sob certas condições de regularidade, a variável aleatória $-2 . \ell n \lambda$, onde $\lambda$ é dada por (7), tem uma distribuiçăo que se aproxima da variável $\mathrm{x}^{2}$, quando $\mathrm{n}$ é finito, com graus de liberdade iguais ao número de pa râmetros que sấo determinados pela hipótese $H_{0}$ "[8], ou seja, os graus de liberdade para o teste sâo iguais ao do teste de qui -quadrado.

$$
-2 \ln \lambda=-2 \cdot \sum_{i=1}^{k} x_{i} \text { \&n }\left[\frac{e_{j}}{x_{i}}\right]=2 \cdot \sum_{i=1}^{k} x_{i} \text { \&n }\left[\frac{e_{j}}{x_{i}}\right]^{-1}
$$

Logo:

$$
G=2 \cdot \sum^{k} x_{i} \text { en }\left[\frac{x_{i}}{e_{i}}\right]
$$

Esta estatística é pouco conhecida e utilizada devido a ser mais trabalhoso aplicar um teste utilizando logarítmos, mas com o uso do computador torna-se mais fácil sua aplicaçấo.

Em geral, G será numericamente similar a $x^{2}$, sendo que o emprego do teste $G$ requer nenhuma sofisticaçăo matemática especial e tem algumas vantagens consideráveis em projetos mais complexos sobre os testes convencionais que utilizam a estatística $\mathrm{x}^{2}$, pois permite-certos tipos de análises mais detalhadas que são possíveis usando-se a estatística de qui-quadrado [13].

Mesmo com o uso de logaritmos para o teste G, este muitas vezes requer muito menos esforço computacional do que é requerido para o teste de $x^{2}$, isto é verdadeiro para alguns testes [13].

Portanto, pode-se empregar o teste G, sempre que puder ser utilizado um computador, pois os resultados numéricos entre $\mathrm{G}$ e $\mathrm{x}^{2}$ podem diferir em alguns casos, sugerindo uma análise mais cuidadosa dos dados coletados [13].

\section{2- ORIGEM DA ESTATÍSTICA QUI-QUADRADO A PARTIR DA ESTATÍSTICA G}

Em 1960, Karl Pearson propôs o seguinte teste estatístico, o qual é funçáo dos quadrados dos desvios das frequências observadas, com relação aos valores esperados respectivos.

$$
x^{2}=\sum_{i=1}^{k} \frac{\left(x_{i}-e_{i}\right)^{2}}{e_{i}}
$$


Pode-se demonstrar a origem da estatística $\mathrm{X}^{2}$ a partir da estatística $G$, da seguinte maneira:

Considerando-se a Estatísitca G, a Eq. (11), e fazendo-se $y_{i}=x_{i}-e_{i}$, a diferença entre as frequências observadas e esperadas na i-ésima cela, tem-se:

$$
G=2 \Sigma\left(e_{i}+y_{i}\right) \ln \frac{e_{i}+y_{i}}{e_{i}}=2 \Sigma\left(e_{i}+y_{i}\right) \ln \left[1+\frac{y_{i}}{e_{i}}\right]
$$

Expandindo In $\left[1+\frac{y_{i}}{e_{i}}\right]$ em Série de Taylor em torno de $\mathbf{x}=\Phi$ obtém-se:

$$
G=2 \Sigma\left(y_{i}+e_{i}\right) \cdot\left[\frac{y_{i}}{e_{i}}-\frac{1}{2}\left[\frac{y_{i}}{e_{i}}\right]^{2}+\frac{1}{3}\left[\frac{y_{i}}{e_{i}}\right]^{3}-\ldots\right]
$$

Efetuando, resulta:

$\mathrm{G}=\Sigma \frac{\mathrm{y}_{i}}{\mathrm{e}_{\mathrm{i}}}-\frac{1}{3} \Sigma \frac{\mathrm{y}_{\mathrm{i}}{ }^{3}}{\mathrm{e}_{\mathrm{i}}}+\ldots \ldots \ldots$

A variável y é uma variável binomial com média $\mu_{i}=$ $\mathrm{n} \cdot \mathrm{p}_{i}=\mathrm{e}_{i}$ e variância $\sigma_{i}{ }^{2}=n p_{i}(1-\mathrm{p}) ;$ consequentemente, $a$ variável $\frac{y_{i}}{e_{i}}$ pode ser expressa na forma:

$$
\frac{y_{i}}{e_{i}}=\frac{x_{i}-e_{i}}{e_{i}}=\frac{x_{i}-\mu_{i}}{\sigma_{i}} \sqrt{\left(1-p_{i}\right) / n} p_{i}
$$

Do Teorema "Se X é normalmente distribuída com média e variância $\sigma^{2}$ e uma amostra aleatória de tamanho n é retirada, entâo a média amostral $\bar{x}$ será normalmente distribuída com média $\mu$ e variâcia $\frac{\sigma^{2}}{n}$ "[8], a variável $\left(x_{i}-\mu_{i}\right) / \sigma_{i}$ tem distribuiçăo aproxi-

mada à de uma variável normal padrăo quando $n \rightarrow \infty$. Sendo assim o fator da raiz quadrada da Eq. (14) se aproxima de zero na mesma taxa que $1 / \sqrt{n}$. Então, para $n$ muito grande $y_{i} / e_{i}$ será muito pequeno e da ordem de $1 / \sqrt{n}$. Como consequência os termos sucessivos na expansăo anterior serắo da ordem de $1 / \sqrt{n}$ vezes 0 termos precedente.

0 resultado aproximado de -2 en $\lambda$ para amostras grandes é dado pelo 12 termos da direita da Eq. (13). Então:

$$
-2 \ln \lambda \cong \sum \frac{y_{i}^{2}}{e_{i}}=\sum_{i=1}^{k} \frac{\left(x_{i}-e_{i}\right)^{2}}{e_{i}}
$$

Como -2 थก $\lambda$ possui distribuiçăo $x^{2}$ aproximada, entăo 0 termo da direita da Eq. (15) também possui distribuiçăo $\mathrm{x}^{2}$.

3- CONCLUSÕES :

Através da literatura citada chegou-se as seguintes conclusôes :

- Em projetos mais complexos a aplicação da estatística G tem algumas vantagens em relaçăo a aplicaçăo da estatística $\mathrm{x}^{2}$, pois possibilita uma análise mais detalhada dos dados;

- A estatística G é pouco utilizada devido, principalmen te, a falta de informaçôes sobre o assunto causada pela escassa li 
teratura e também pela maior dificuldade de cálculos pelo emprego de logarítmos, o que no momento não é mais problema com o uso do computador.

\section{4- REFERÊNCIAS BIBLIOGRÁFICAS}

01. AYRES, M. \& AYRES JUNIOR, M Aplicações Estatísticas em Basic. Săo Paulo, Mc Graw-Hill, 1987.

02. BIS $\overline{\mathrm{HOP}}, \mathrm{Y}$. M. et alii. Discrete Multivariate Analysis: Theory and Practice. The mit Press, 1975.

03. CHRISTMANN, Raul U. Estatística Aplicada. 2@ ed. Săo Pau10, Edgard Blucher Ltda, 1978.

04. CONOVER, W. J. Practical Nonparametric Statistic. New York, John Wiley \& Sons, 1971.

05. COSTA NETO, Pedro L. de O. Estatística. Săo Paulo, Edgard Blucher Ltda, 1977.

06. FONSECA, Jairo S. da, MARTINS, Gilberto de A. Curso de Estatís tica. 3a ed. São Paulo, Atlas, 1982.

07. GoODMAN, Richard. Estatística. Săo Paulo, Livraria Editora Pioneira, 1965.

08. HOEL, Paul G. Estatística Matemática. 4a ed. Rio de Janeiro, Guanabara Dois S.A., 1980.

09. MENDENHALL, William. Probabilidade e Estatística. Rio de Janei ro, Campus, 1985, v. 2 .

10. RODRIGUES, Aroldo. A Pesquisa Experimental em Psicologia e Educaçăo. 2@ ed. Rio de Janeiro, Vozes, 1976.

11. SCREMIN, Marcos A. A. Comparaçăo Entre os Testes G e Qui-Quadrado Através de um Programa Computacional. Monografia do Curso de Pós-Graduaçăo em Métodos Quantitativos, Universida de Federal de Santa Maria, Santa Maria-RS, 1989.

12. SIEGEL, Sidney. Estatística Năo-paramétrica. São Paulo, McGraw-Hill do Brasil, Ltda, 1975.

13. SOKAL, R. and ROHLF, F. J. BIOMETRY. San Francisco, Freeman and Company, 1969 .

14. STEVENSON, Willian J. Estatística Aplicada à Administraçăo. Sắo Paulo, Harper \& Row do Brasil Ltda, 1981.

15. YAMANE, Taro. Estatística. 3 ed. México, Harla, 1974.

Recebido em outubro, 1990; aceito em abril, 1991. 
